\title{
EFECTO PROTECTOR DEL Petroselinum crispum (MILL.) A.W. HILL (PEREJIL) FRENTE A LA HEPATOTOXICIDAD CRÓNICA INDUCIDA CON ETANOL EN RATAS ALBINAS Holtzman
}

\author{
PROTECTIVE EFFECT OF Petroselinum crispum (MILL.) A.W. HILL (PEREJIL) AGAINST \\ ETHANOL-INDUCED CHRONIC HEPATOTOXICITY IN RATS Holtzman \\ Miraval Edgar', Obando Diana', Lozano Oscar', Vela Manuel', Jurupe Hilda ${ }^{12}$, Herencia Vilma 1,2
}

\section{RESUMEN}

Objetivos: Evaluar el efecto hepatoprotector del extracto hidroalcohólico de Petroselinum crispum (Mill.) A.W. Hill (PEREJIL) sobre la inducción crónica de hepatotoxicidad con etanol en ratas albinas Holtzman jovenes. Métodos: Estudio transversal, analítico y experimental. Se realizó inducción crónica de hepatotoxicidad con etanol al $20 \%$ por 3 meses. Los grupos fueron: Blanco, Control Negativo recibió etanol $20 \%$, Control Positivo etanol $20 \%$ y silimarina $25 \mathrm{mg} / \mathrm{kg}$ y Tratamiento recibió etanol $20 \%$ y extracto hidroalcohólico Petroselinum crispum $150 \mathrm{mg} / \mathrm{kg}$. Se realizaron exámenes bioquímicos (sangre) y anatomopatológicos (hígado). Se utilizaron las pruebas estadísticas de ANOVA y Tukey; se consideró $p<0,05$ como significativo e intervalo de confianza 95\%. Resultados: Las medias de los niveles bioquímicos de ALT, AST, Bilirrubinas totales y GGT para el grupo tratamiento fueron de $51.76 \pm 16.15,112.86 \pm 56.16,0.26 \pm 0.07$ y $0.66 \pm 0.14$. Mientras que las medias de los niveles bioquímicos de ALT, AST, Bilirrubinas totales y GGT para el grupo control positivo fueron de $24.55 \pm 4.21,44.26 \pm 3.85,0.24 \pm 0.06$ y $1.32 \pm 0.62$. La prueba post hoc Tukey para la GGT mostró diferencias entre el grupo control positivo y grupo tratamiento $(p<0,01)$. El estudio anatomopatológico no mostró cambios relacionados a esteatohepatitis en todos los grupos. Conclusión: El extracto hidroalcohólico de Petroselinum crispum (Mill.) A.W. Hill con nula evidencia hepatoprotetora sobre la inducción crónica de hepatotoxicidad con etanol a las dosis evaluadas. Son necesarios más estudios.

Palabras clave: Petroselinum crispum; Perejil; Hepatotoxicidad; Hepatoprotector. (fuente: DeCS BIREME)

\begin{abstract}
Objective: To evaluate the hepatoprotective effect of the hydroalcoholic extract of Petroselinum crispum (Mill.) A.W. Hill (PEREJIL) on the chronic induction of hepatotoxicity with ethanol in young albino rats Holtzman. Methods: Cross-sectional, analytical and experimental study. Chronic hepatotoxicity was induced with ethanol $20 \%$ for 3 months. The groups were: White, Control Negative received ethanol $20 \%$, Control Positive ethanol $20 \%$ and silymarin $25 \mathrm{mg} / \mathrm{kg}$ and Treatment received ethanol $20 \%$ and hydroalcoholic extract Petroselinum crispum $150 \mathrm{mg} / \mathrm{kg}$. Biochemical (blood) and anatomopathological (liver) tests were performed. Statistical tests of ANOVA and Tukey were used; $\mathrm{P}<0.05$ was considered significant and $95 \%$ confidence interval. Results: Mean of the biochemical levels of ALT, AST, total bilirubin and GGT for the treatment group were $51.76 \pm 16.15,112.86$ $\pm 56.16,0.26 \pm 0.07$ and $0.66 \pm 0.14$. While the means of the biochemical levels of ALT, AST, total bilirubin and GGT for the positive control group were $24.55 \pm 4.21,44.26 \pm 3.85,0.24 \pm 0.06$ and $1.32 \pm 0.62$. The Tukey post hoc test for GGT showed differences between the positive control group and the treatment group $(p<0.01)$. The anatomopathological study showed no changes related to steatohepatitis in all groups. Conclusions: The hydroalcoholic extract of Petroselinum crispum (Mill.) A.W. Hill had no hepatoprotective effect on the chronic induction of hepatotoxicity with ethanol at the doses evaluated. More studies are needed.
\end{abstract}

Key words: Petroselinum crispum; Perejil; Hepatotoxicity; Hepatoprotective. (source: MeSH NLM)

Facultad de Medicina Humana, Universidad Ricardo Palma.

${ }^{2}$ Cátedra de Farmacología, FAMURP.

Correspondencia: Herencia Vilma. Dirección: INICIB, Facultad de Medicina Humana, Edificio I. 2do piso. Avenida Benavides 5440, Surco. Lima, Perú. Teléfono: 708-0000 Correo : vilma.herencia@gmail.com

Citar como: Miraval Edgar, Obando Diana, Lozano Oscar, Vela Manuel, Jurupe Hilda, Herencia Vilma. Efecto protector del Petroselinum crispum (Mill.) A.W. Hill (perejil) frente a la hepatotoxicidad crónica inducida con etanol en ratas albinas holtzman [Artículo Original]. Rev. Fac. Med. Hum. 2016;16(3):21-29. DOI 10.25176/RFMH.v16.n3.648 


\section{INTRODUCCIÓN}

A nivel mundial la cirrosis hepática asociada al consumo de alcohol es una de las diez causas más frecuentes de muerte en el mundo occidental y en nuestro país ocupando un lugar prominente dentro de las causas de morbiletalidad ${ }^{1,2}$. Cada año debido al consumo nocivo de alcohol se producen 3,3 millones de muertes en el mundo 3 . En el Perú, el abuso y la dependencia al alcohol figuran como la tercera causa de pérdidas por muerte prematura o discapacidad, carga que es superada sólo por las causadas por la neumonía y los accidentes de tránsito. En términos económicos, según DEVIDA, la problemática del alcohol le genera al Perú pérdidas de al menos 245 millones de dólares anualmente. El consumo crónico de bebidas alcohólicas, es más frecuente que los mencionados sobre todo en poblaciones jóvenes y adultas indistintamente del sexo, factor principal que predispone a cirrosis que es la quinta causa de muerte de hepatopatía y un problema de salud pública en nuestro país 4,5 . Dentro de las medidas para controlar este problema de salud pública, la medicina tradicional es una alternativa con gran potencial de ayuda, especialmente en regiones donde el sistema de salud es insuficiente para la demanda de la problemática de salud ${ }^{6-10}$. Una de las principales plantas utilizadas en medicina tradicional y accesible son de la familia Apiaceae o umbelíferas.

Petroselinum crispum (Mill.) A.W. Hill (perejil); es una planta herbácea bienal aromática perteneciente a la familia Apiaceae o umbelíferas; perenne de corta duración, muy ramificada, de color verde y tallo cilíndrico, conocida comúnmente como Perejil. Esta especie vegetal se cultivaba en grandes cantidades ya desde la época medieval, se distribuye ampliamente por todo el mundo y generalmente se cultiva para ser usada como condimento ${ }^{11,12}$. Dentro de las propiedades terapéuticas atribuidas al perejil gracias que los componentes característicos de flavonoides (apína, luteolina, apigenina y algunos glucósidos), aceites esenciales (apiol y miristicina), cumarinas (bergapteno, imperatorina, xantotoxina, trioxaleno y angelicina), así como vitaminas $\mathrm{C}$ y $\mathrm{E}_{\text {, }}$ mencionando además que es la fuente más rica en vitamina $A$.,vitaminas $C$ y $E^{13,14}$, algunas vitaminas del complejo B, calcio, hierro, fósforo y azufre, además alta concentración de ácido petroselínico (isómero del ácido oleico), furanocumarinas, oleorresinas, proteínas, carbohidratos y taninos ${ }^{15}$; con todo ello también se registran reportes de su actividad antioxidante ${ }^{14-16}$, se le confiere propiedades laxantes, atribuibles a la presencia de algunos aceites volátiles que están más concentrados en las semillas que en tallos $u$ hojas, gran cantidad de compuestos fotoquímicos o fitonutrimentos, y es posible que muchos de ellos impidan que las células normales y sanas se vuelvan cancerosas, propiedades cataplasmaticas para muchos problemas menores, como esguinces, cortaduras, picaduras y quemaduras, propiedades diuréticas, usado como medicina empírica para disminuir la glucosa en la sangre ${ }^{16-19}$, y se ha demostrado su efectividad en el tratamiento de los trastornos gastrointestinales mediante estudios con ratas ${ }^{19}$. Agregar que el perejil es usado con fines terapéuticos, como relajante muscular, diurético, carminativo, expectorante, reumatoide, antibronquial,

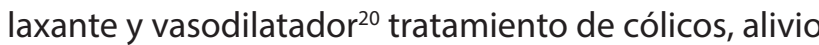
de la inflamación de la vejiga, para la interrupción de la lactancia, reducción de los dolores de las encías y en el tratamiento de las enfermedades de la piel; así mismo, presenta actividad anticoagulante ${ }^{21}$ éstas son propiedades atribuidas al uso tradicional y ameritaría de estudios científicos.

Esta investigación promete aportes para futuros proyectos pre-clínicos y clínicos cuya búsqueda sea el uso de plantas medicinales como el Petroselinum crispum entre otras en la necesidad de encontrar las propiedades terapéuticas como hepatoprotectora, antioxidante, antiinflamatoria, entre otras y permitiría recomendarse como tratamiento o coadyuvante tanto en la profilaxis y tratamiento de enfermedades hepáticas entre otras.

A pesar de esto, no hay estudios acerca de la acción de Petroselinum crispum (Mill.) A.W. Hill (perejil) sobre su actividad regenerativa, antioxidante, antiinflamatoria a nivel hepático. Por lo expuesto el estudio busca evaluar el efecto hepatoprotector del extracto hidroalcoholico de Petroselinum crispum (Mill.) A.W. Hill (perejil) frente a la hepatotoxicidad crónica inducida por etanol al $20 \%$ en ratas albinas prometiendo aportes para futuros proyectos pre-clínicos y clínicos.

\section{MÉTODOS}

Se realizó un estudio tipo analítico, experimental, transversal y prospectivo.

Se recolecto la materia vegetal elegida Petroselinum crispum (Mill.) A.W. Hill (perejil), enviando la muestra para su respectiva posición taxonómica, según el sistema de clasificación de Cronquist (1998) realizado en el Museo de Historia Natural - UNMSM Se obtiene una muestra completa, de hojas y tallos; se dejaron 
al secado a $40^{\circ} \mathrm{C}$ en horno por 3 días y luego a temperatura ambiente bajo sombra por 3 días. Se trituraron manualmente con ayuda de un rodillo, la molienda se realizó en un mortero a mano.

Luego el material vegetal ya en polvo y bajo condiciones de calidad de la muestra según las normas establecidas por farmacopeas, se colocó en una solución hidroalcoholica al 75\% macerándose por 7 días, luego se filtró y llevando al secado por 10 días a temperatura y humedad ambiental obtenido el extracto seco se preparó una solución usando como diluyente agua/tween 801:1.

El modelo experimental farmacológico: Se utilizó 40 ratas albinas sp. Holtzman, jóvenes de 01 mes de edad, cuyos pesos estaban comprendidos entre 100 a $150 \mathrm{~g}$. distribuidas de manera aleatoria en cuatro grupos, grupo blanco, grupo control negativo, grupo control positivo y grupo tratamiento, de 10 ratas cada uno. Manteniéndolas a temperatura y humedad ambiental con acceso libre al aire alimentación balanceada y agua ad libitum por 15 días para su aclimatación en el Laboratorio de Farmacología de la Facultad de Medicina de la URP.

Inducción de la Hepatoxicidad: La inducción de la toxicidad hepática por consumo crónico de alcohol $20 \%$, durante 90 días, en este tiempo las ratas recibieron alcohol ad libitum en vez de agua además de la alimentación balanceada.

Durante el proceso de inducción el día 60, se inició con el tratamiento, tal como sigue: Grupo Blanco (GB); solo recibió agua ad libitum. Grupo Control Negativo (CN); solución de alcohol 20\%, ad libitum. Grupo Control Positivo (CP); solución de alcohol 20\%, ad libitum + Silimarina dosis $25 \mathrm{mg} / \mathrm{kg}$. Grupo Tratamiento (GT); solución de alcohol $20 \%$, ad libitum + solución de extracto hidroalcoholico de Petroselinum crispum (perejil) dosis $150 \mathrm{mg} / \mathrm{kg}$. Se administró los tratamientos por 30 días, vía intragástrica. El día 90 se inició con el análisis bioquímico y patológico; las ratas fueron anestesiadas con Pentobarbital sódico $30 \mathrm{mg} /$ kg. La extrajo sangre por punción cardiaca colocando las muestras de sangre en frascos heparinizados y posteriormente por laparotomía se extrajo el hígado de cada rata colocándolos en frascos con $40 \mathrm{~mL}$. de una solución de formol (formaldehido 40\%) tamponado con baffer fosfato ${ }^{1: 9-14}$.

Se realizaron las siguientes determinaciones en suero sanguíneo de las ratas; glucosa (GLU), alanina aminotransferasa (ALT), aspartato aminotransferasa (AST), fosfatasa alcalina (FA), gamma glutamil transferasa (GGT), proteínas totales (PT), triglicéridos
(TG), colesterol (CHO), bilirrubinas totales (BILT), creatinina (Crea) y Urea. Al mismo tiempo también se hizo una determinación de hemograma, con el fin de detectar si los parámetros hemáticos varían con los diferentes tratamientos administrados ${ }^{14}$.

Para el estudio histopatológico se practicó cortes de $0,5 \times 1,0 \mathrm{~cm}$ de espesor, que fueron fijados en formol neutro al 10\%, los cuales fueron seccionados para inclusión en cortes de $2 \mathrm{~mm}$ de espesor; posteriormente se efectuó cortes con micrótomo, en un espesor de 3um, para luego ser coloreados con HE (hematoxilina-eosina) y revisados al microscopio óptico, para la identificación de signos de congestión vascular, infiltrados inflamatorios, necrosis del parénquima y regeneración posnecrótica ${ }^{21}$.

Los resultados obtenidos se colocaron en una base de datos realizada en Microsoft Excel 2010 y procesados en el programa estadístico SPSS ${ }^{22}$. Se utilizó la prueba de ANOVA de 1 cola y la prueba post hoc de Tukey, para las comparaciones entre los grupos. Se trabajó a una significancia estadística de un valor de $p<0.05$ y un intervalo de confianza al 95\%.

\section{RESULTADOS}

La evaluación del efecto hepatoprotector del Petroselinum crispum (perejil) frente a la hepatotoxicidad crónica inducida con etanol al 20\% en ratas albinas Holtzman obtuvimos los siguientes resultados:

Posterior a la inducción de hepatotoxicidad (día 21) y antes de iniciar el tratamiento (día 60) a los grupos CP y GT, se obtuvo una masa corporal comprendida entre $185 \mathrm{~g}$ y $224 \mathrm{~g}$ con un promedio de $205.6 \mathrm{~g}$ y al término de la inducción tuvieron un peso corporal final entre $210 \mathrm{~g}$ y $245 \mathrm{~g}$ con un promedio de $230.5 \mathrm{~g}$. La ganancia promedio de masa corporal en gramos de las ratas al final del tratamiento (Tabla1).

Tabla 1. Ganancia de Peso al final del experimento.

\begin{tabular}{cccc}
$\begin{array}{c}\text { Grupo Blanco } \\
\text { (GB) }\end{array}$ & $\begin{array}{c}\text { Control } \\
\text { Negativo } \\
\text { (CN) }\end{array}$ & $\begin{array}{c}\text { Control } \\
\text { Positivo (CP) }\end{array}$ & $\begin{array}{c}\text { Tratamiento } \\
\text { (GT) }\end{array}$ \\
\hline $20.60 \pm 8.34$ & $23.50 \pm 6.70$ & $24.10 \pm 10.79$ & $30.80 \pm 7.06$ \\
& Ganancia de Peso (g) \\
\hline
\end{tabular}

Estos valores de medias no obtuvieron diferencias estadísticas significativas a un $\mathrm{p}<0.05$, sin embargo el GT tuvo mayor ganancia de masa corporal en gramos que los demás grupos. 
Los parámetros bioquímicos del suero sanguíneo de las ratas (Tabla 2). Los valores de referencia están basados en los exámenes de laboratorio que realizó las pruebas enzimáticas.

Tabla 2. Valores promedio de Bioquímica Sanguínea al final del tratamiento.

\begin{tabular}{|c|c|c|c|c|}
\hline & $\begin{array}{c}\text { Grupo Blanco } \\
\text { (GB) }\end{array}$ & $\begin{array}{l}\text { Control Negativo } \\
\text { (CN) }\end{array}$ & $\begin{array}{l}\text { Control Positivo } \\
\text { (CP) }\end{array}$ & $\begin{array}{l}\text { Tratamiento } \\
\text { (GT) }\end{array}$ \\
\hline Alalnina aminotransferasa(U/L) & $52.19 \pm 16.55$ & $55.37 \pm 15.36$ & $24.55 \pm 4.21$ & $51.76 \pm 16.15$ \\
\hline Aspartato aminotransferasa(U/L) & $61.87 \pm 16.81$ & $61.29 \pm 18.24$ & $44.26 \pm 3.85$ & $112.86 \pm 56.16$ \\
\hline Fosfatasa alcalina (U/L) & $59.15 \pm 14.90$ & $62.10 \pm 15.80$ & $71.28 \pm 21.35$ & $52.10 \pm 6.70$ \\
\hline Proteinas Totales (g/dl) & $6.88 \pm 0.52$ & $6.99 \pm 0.51$ & $6.56 \pm 0.34$ & $6.78 \pm 0.40$ \\
\hline Gamma glutariltransferasa(U/L) & $0.88 \pm 0.28$ & $1.04 \pm 0.34$ & $1.32 \pm 0.62$ & $0.66 \pm 0.14$ \\
\hline Triglicéridos(mg/dl) & $59.01 \pm 7.42$ & $58.57 \pm 7.01$ & $68.72 \pm 3.67$ & $69.64 \pm 5.06$ \\
\hline Colesterol(mg/dl) & $85.40 \pm 8.79$ & $91.62 \pm 9.44$ & $85.71 \pm 5.46$ & $83.35 \pm 6.62$ \\
\hline Billirrubinas totales(mg/dl) & $0.16 \pm 0.03$ & $0.23 \pm 0.07$ & $0.24 \pm 0.06$ & $0.26 \pm 0.07$ \\
\hline Glucosa(mg/dl) & $153.94 \pm 30.75$ & $149.04 \pm 34.71$ & $160.13 \pm 31.11$ & $137.43 \pm 11.08$ \\
\hline Urea(mg/dl) & $60.24 \pm 5.41$ & $59.32 \pm 3.99$ & $59.76 \pm 4.35$ & $59.69 \pm 5.18$ \\
\hline Creatinina(mg/dl) & $0.67 \pm 0.04$ & $0.64 \pm 0.05$ & $0.65 \pm 0.05$ & $0.67 \pm 0.05$ \\
\hline
\end{tabular}

En relación a la bioquímica se encontraron diferencias estadísticas significativas con el test ANOVA para la ALT, AST, GGT, TG y BILT ( $p<0.05)$.

Utilizando pruebas post-hoc (Tukey) se observó diferencias significativas en los valores de las medias de ALT para el Control Positivo con los grupos Blanco $(p<0.01)$, Control Negativo $(p<0.001)$ y el Grupo Tratamiento $(p<0,01)$. También se encontraron diferencias para las medias de AST del Grupo Tratamiento con el Control Blanco $(p<0.01)$, Control Negativo $(p<0.01)$ y Control Positivo $(p<0.001)$. En cuanto a las BILT se encontró diferencias del grupo Control Blanco con el Control Positivo $(p<0.05)$ y con el Grupo Tratamiento $(p<0.01)$.

En la GGT se observó diferencias significativas entre el Control Positivo con Control Negativo $(p<0.05)$ y entre el Grupo Tratamiento con el Control Positivo $(p<0.01)$.
Por otra parte, en los TG se encontró diferencia entre los grupos Control Positivo con el Control Blanco $(p<0,01)$ y Control Negativo $(p<0,01)$, así como también para el GT con el Control Blanco $(p<0,01)$ y Control Negativo $(p<0,01)$.

Los valores plasmáticos de glucosa, fosfatasa alcalina, proteínas totales, colesterol, urea y creatinina no resultaron estadísticamente significativos a un $\mathrm{p}<0.05$. Sin embargo rescatar el valor de glucosa en el grupo CP obtuvo mayor promedio que los demás y lo mismo ocurrió en el CN para el colesterol que obtuvo mayor promedio que los otros. Los parámetros sanguíneos (Tabla 3). 
Tabla 3. Valores promedio del Hemograma al final del tratamiento.

\begin{tabular}{|c|c|c|c|c|}
\hline & $\begin{array}{c}\text { Grupo Blanco } \\
\text { (GB) }\end{array}$ & $\begin{array}{l}\text { Control Negativo } \\
\text { (CN) }\end{array}$ & $\begin{array}{c}\text { Control Positivo } \\
\text { (CP) }\end{array}$ & $\begin{array}{c}\text { Tratamiento } \\
\text { (GT) }\end{array}$ \\
\hline $\begin{array}{l}\text { Glóbulos blancos } \\
\left(10^{3} / \mathrm{ul}\right) \mathrm{VN}=2.8-8.2\end{array}$ & $1.35 \pm 0.34$ & $1.39 \pm 0.39$ & $1.51 \pm 0.68$ & $1.59 \pm 0.19$ \\
\hline $\begin{array}{l}\% \text { Abastonados } \\
\text { VN=0-2 }\end{array}$ & $5.60 \pm 1.90$ & $5.20 \pm 1.48$ & $4.30 \pm 1.64$ & $4.20 \pm 1.87$ \\
\hline$\%$ Segmentados & $45.50 \pm 6.40$ & $33.40 \pm 11.96$ & $26.50 \pm 6.47$ & $38.50 \pm 11.89$ \\
\hline$\%$ Linfocitos & $43.90 \pm 6.30$ & $56.50 \pm 14.77$ & $61.70 \pm 7.32$ & $50.40 \pm 11.40$ \\
\hline \%Eosinófilos & $4.20 \pm 2.35$ & $4.40 \pm 2.80$ & $6.60 \pm 1.90$ & $6.40 \pm 0.97$ \\
\hline \%Monocitos & $1.00 \pm 0.82$ & $0.70 \pm 0.82$ & $0.90 \pm 0.57$ & $0.70 \pm 0.67$ \\
\hline Glóbulos rojos(10\%/ul) & $5.12 \pm 0.99$ & $4.75 \pm 1.28$ & $5.82 \pm 1.37$ & $7.41 \pm 1.76$ \\
\hline Hemoglobina(g/dl) & $9.66 \pm 2.05$ & $9.88 \pm 2.32$ & $11.93 \pm 2.61$ & $12.62 \pm 2.94$ \\
\hline $\begin{array}{l}\text { \%Hematocrito } \\
\mathrm{VN}=31.4-39.4\end{array}$ & $33.51 \pm 5.52$ & $29.91 \pm 6.64$ & $34.28 \pm 7.18$ & $40.00 \pm 9.98$ \\
\hline Plaquetas $\left(10^{3} /\right.$ ul) & $481.40 \pm 53.92$ & $474.50 \pm 50.08$ & $379.30 \pm 65.91$ & $358.20 \pm 76.88$ \\
\hline
\end{tabular}

En la evaluación del hemograma con la prueba ANOVA encontró significancia estadística en los parámetros porcentuales (\%) de segmentados, linfocitos, eosinófilos, glóbulos rojos por $\mathrm{mm} 3$, hemoglobina, hematocrito y plaquetas a un $\mathrm{p}<0.05$.

En cuanto al estudio anatomopatológico: El grupo CN y CP se pudo evidenciar la presencia de leve infiltrado inflamatorio linfocitario y discreta congestión vascular (Figura 1 y 2). En el GT se observó leve congestión vascular al igual que leves infiltrados inflamatorios linfocitarios, con discreta hiperplasia de células de Kuffer (Figura 3).
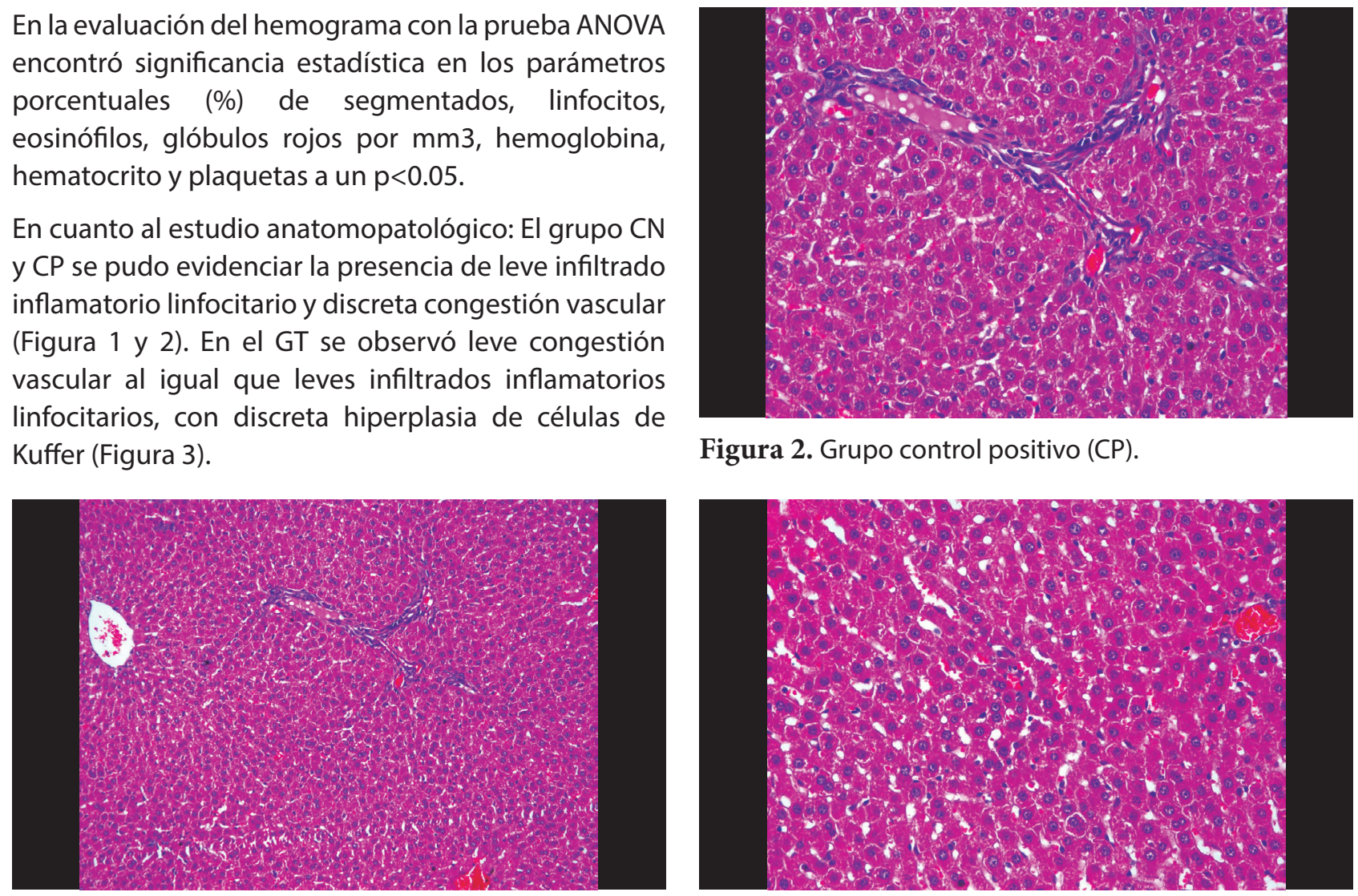

Figura 2. Grupo control positivo (CP).

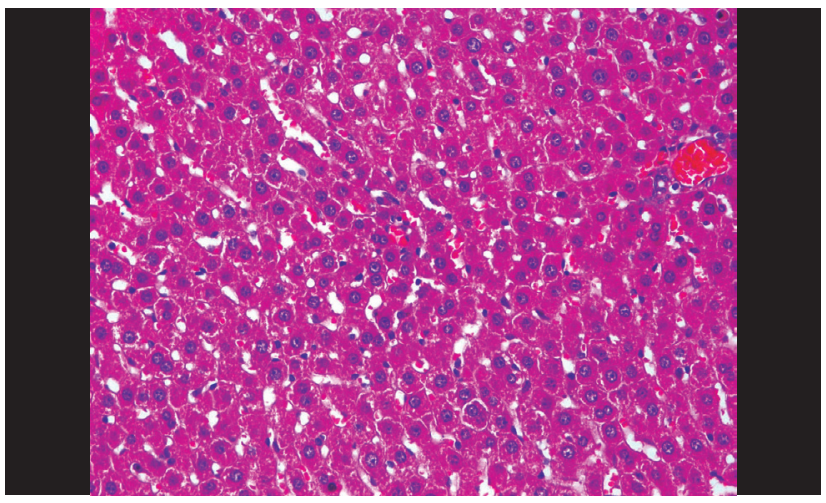

Figura 1. Grupo control negativo (CN).

Figura 3. Grupo tratamiento (GT). 


\section{DISCUSIÓN}

Investigar sobre plantas medicinales y sus diversos efectos farmacológicos es en nuestra región unos de los pilares importantes en la búsqueda de alternativas y opciones terapéuticas en medicina y que a su vez aprovechen la base tecnológica y productiva local, es decir, las plantas nativas y de uso común, ya que son ampliamente usadas por la mayoría de nuestra población tanto urbana como rural. Además, la riqueza de las plantas medicinales es muy amplia y está enmarcada dentro de más de 1400 especies de plantas medicinales conocidas por las poblaciones locales, de las cuales un gran porcentaje se presenta en la región andina, tal como lo recalca Brack E. ${ }^{6}$. Es motivo suficiente para rescatar a la fitoterapia e integrarla como parte de las estrategias de los sistemas terapéuticos y que es fundamental la inversión en este sector, para explotar el potencial que tiene ${ }^{7}$.

Las propiedades que se le atribuyen al Petroselinum crispum (Mill.) A.W. Hill (perejil) se debe a los componentes químicos que posee y que se han reportado como flavonoides (apíina, luteolina, apigenina y algunos glucósidos), aceite esencial (apiol y miristicina), cumarinas (bergapteno, imperatorina, xantotoxina, trioxaleno y angelicina), así como vitaminas $C$ y $E$, algunas vitaminas del complejo $B$, calcio, hierro, fósforo, azufre, alta concentración de ácido petroselínico, furanocumarinas, oleorresinas, proteínas, carbohidratos y taninos. De todos estos rescatar a los flavonoides, los cuales le confieren propiedades diuréticas, antioxidantes, emenagogas (estimula la menstruación), entre otras ${ }^{8}$.

Un ejemplo claro de esto, es el trabajo de Nagamani et al. al demostrar, por estudios histopatológicos, protección con Vimliv, cuyo componente principal son los flavonoides, tanto en hígado como en riñones de ratas albinas wistar frente a la toxicidad por etanol a dosis de $3 \mathrm{mg} / \mathrm{kg}$ por 35 días $^{9}$. Otro ejemplo seria el trabajo de Selema et al. que corrobora el efecto hepatoprotector de flavonoide astilbina, teniendo como base niveles menores de transaminasa glutámica pirúvica (ALP) y fosfolipasa A2 en el grupo tratamiento con respecto al grupo blanco, grupo de Tetracloruro $\mathrm{CCl} 40,001 \mathrm{ml} / \mathrm{g}$ como control negativo y grupo con dimetilsulfoxido DMSO un fitofármaco como control positivo ${ }^{10}$.

Cabe mencionar que al igual que los flavonoides, los compuestos que hayan demostrado tener efecto antioxidante son importantes para detener el avance de enfermedades en las cuales esté vinculado el estrés oxidativo en mayor o menor grado y en específico el daño hepático inducido por alcohol ya que está relacionado, al menos en parte, a una producción elevada de radicales libres de oxígeno y/o a un descenso de los niveles de antioxidantes ${ }^{11,12}$.

Los resultados bioquímicos obtenidos en el presente trabajo experimental refieren ausencia de disminución de los niveles de AST en el GT, en comparación con los demás grupos inclusive el grupo CP obtuvo cifras promedio más bajas que todos, así como también en los niveles de ALP. Esto puede deberse a que la administración crónica (1 mes) de Silimarina tendría más efecto hepatoprotector que el Petroselinium crispum a la dosis de $150 \mathrm{mg} / \mathrm{kg}$. Sin embargo, se observó ligera disminución de los niveles de GGT en el GT. Por otro lado la bioquímica sanguínea en general no manifestó alteraciones que sobrepasen los niveles normales de referencia vistos en trabajos pasados en Rattus novergicus ${ }^{11-14}$.

Cabe resaltar que en todos los grupos experimentales las medias por debajo de los valores normales en cuanto a los glóbulos blancos, presentando leucopenia y además las medias para los glóbulos rojos y hemoglobina de los grupos GB y $\mathrm{CN}$ que se encuentran por debajo de los valores normales presentando anemia. Otro parámetro que tuvo valores bajos fueron las plaquetas en el grupo CP y GT, pero sin llegar a causar plaquetopenia. A su vez los parámetros hemáticos restantes no presentaron niveles fuera de lo normal en cuanto a valores de referencia obtenidos de artículos que elaboraron parámetros de referencia hemáticos para Rattus Norvegicus ${ }^{13,15,16}$.

La inducción de daño hepático que se escogió para nuestro modelo experimental fue modificada del trabajo de la Dra. Aleida Herrera Batista y se eligió la concentración de etanol al $20 \%$ frente al $40 \%$ que propone el artículo para prolongar el tiempo de administración y prever una insuficiencia hepática fulminante. Sin embargo, existen diversos modelos encontrados como la anastomosis porto-cava terminolateral, daño agudo a las pocas horas con azoximetano, tioacetamida intraperitoneal por 3 días consecutivos, D-galactosamina con cambios hepáticos a las $48 \mathrm{~h}^{17}$, administración intraperitoneal de 50, 100, 200 y 400 mg de vitamina E/día, durante 20 días $^{18}$ y el aumento de lípidos sanguíneos con aceites vegetales ${ }^{19}$ por mencionar algunos ejemplos encontrados.

Nuestro trabajo, con una administración de etanol al $20 \%$ ad libitum por un periodo de 90 días, no logró desarrollar cambios histopatológicos significativos que sugieran algún grado esteatosis hepática en las 
ratas de los grupos CN, CP y GT a comparación con la Dra. A Aleida et al. que, a una dosis de etanol de $6 \mathrm{~g} /$ kg de peso corporal por 62 días con 2 tomas diarias, logró observar en los hígados de sus ratas muerte celular por apoptosis y necrosis, infiltrado leucocitario, cuerpos de Mallory y megalomitocondrias por estudio anatomopatológico. Además encontró diferencias estadísticamente significativas en los valores de la Alanina Aminotrasnferasa entre ratas alcohólicas y controles. Por otro lado recalca que la adolescencia en estas ratas las vuelve susceptibles al etanol que en etapas posteriores ${ }^{20}$.

Un contexto parecido se pudo contrastar con el trabajo de Troncozo et al. en el cual obtuvo efecto hepatoprotector del extracto acuoso de perejil $(150 \mathrm{mg} / \mathrm{kg})$ frente a otro hepatotoxico importante como es el paracetamol (200 mg/ kg) diariamente, durante 5 días, demostrado en los hígados de ratas tratadas con paracetamol mostraron por estudio anatomopatológico signos de necrosis entre moderada y severa, en contraste con las ratas tratadas con paracetamol y perejil que permanecían sin alteraciones, con signos de necrosis leve o necrosis moderada acompañados de regeneración celular marcada. Sumado a esto, obtuvieron niveles de Aspartato aminotransferasa y de Alalnina aminotrasnferasa altos y con diferencias estadísticamente significativas al comparar el grupo de inducción de hepatotoxicidad con paracetamol y el tratado con extracto acuso perejil más paracetamol ${ }^{21}$.

Por considerar otros ejemplos más, está el de Ana María Guevara-Vásquez et al. que realizó un trabajo de hepatoprotección del perejil $(150 \mathrm{mg} / \mathrm{kg})$ frente al paracetamol $(1 \mathrm{~g} / \mathrm{kg})$ durante 3 días concluyendo que existe actividad hepatoprotectora al disminuir los niveles plasmáticos de la alanina aminotransferasa de manera significativa en comparación con grupo el control y también por proteger la arquitectura celular de los hepatocitos de las ratas ${ }^{22}$. Otro ejemplo sería mencionar a Acela Inés Arnao-Salas et al. que lograron atenuar la intoxicación con paracetamol a dosis de $250 \mathrm{mg} / \mathrm{kg}$ en ratas hembras, evidenciada por actividades aumentadas de AST, ALT, y GTP, los niveles incrementados de bilirrubina total y disminuidos de proteínas totales que fue atenuada por la administración del extracto acuoso de Smallanthus sonchifolius (Yacón), teniendo como mejor dosis a $200 \mathrm{mg} / \mathrm{kg}^{23}$. También cabe rescatar a Christiam Ochoa et al. que detuvo la hepatotoxicidad del paracetamol a dosis de $200 \mathrm{mg} / \mathrm{kg}$ con extracto acuoso de Peumus boldus a dosis de $80 \mathrm{mg} / \mathrm{kg}, 120 \mathrm{mg} / \mathrm{kg}$ y $160 \mathrm{mg} / \mathrm{kg}$ al frenar el incremento de alanina aminotransferasa y no evidenciar signos de hígado graso por histopatología en sus grupos tratamiento ${ }^{24}$. Y por último mencionar otros trabajos prueban la hepatoprotección de la Mentha piperita, Onicum basilicum y Allium sativum frente a la hepatotoxicidad con paracetamol 25,26 .

El efecto que el Petroselinum crispum y otras plantas han podido ofrecer frente al paracetamol se atribuye en parte a tu potencial antioxidante $e^{27-35}$ y que la toxicidad por este medicamento se basa en generar cantidades de $\mathrm{N}$-acetil p-benzoquinoneimina (NAPQI) capaces de agotar las reservas hepáticas de glutatión, unirse de forma covalente a macromoléculas y producir radicales libres que desarrollarían necrosis hepática en tan solo 12 horas $^{36}$.

Un hepatotoxico de importancia frente al cual también se han realizado estudios de hepatoprotección es el tetracloruro $(\mathrm{CCl} 4)^{17,37}$ y ha sido contrastado frente al efecto del extracto de Taraxacum officinale (diente de león), el cual brindó actividad hepatoprotectora a dosis $200 \mathrm{mg} / \mathrm{kg}$ por 9 días $^{37}$. También se puede rescatar el trabajo de J. Arroyo et.al. que pudo detener el daño hepático por tetracloruro con diferentes dosis de Piper aduncum (matico) dosis 100mg/kg, 200mg/ $\mathrm{kg}$ y $400 \mathrm{mg} / \mathrm{kg}$ los cuales no permitieron el aumento de los niveles de alanina aminotransferasa (ALT) en comparación con el grupo de ratas que solo recibió tetracloruro (CCl4). Otro hepatotoxico hallado en la literatura es el Valproato de sodio a dosis de 500mg/ kg por 7 días con el cual se pudo demostrar que el extracto de Petroselinum crispum a dosis de $200 \mathrm{mg} /$ kg ejerce efecto hepatoprotector ${ }^{38}$.

En nuestro trabajo experimental la escasez de modelos de hepatotoxicidad crónica que permitieran un modelo experimental como marco de referencia fue una limitación, debido a que la literatura consultada en su mayoría realizó trabajos experimentales en periodos cortos de tiempo, es decir, trabajando con intoxicación hepática aguda independiente del hepatotoxico usado 12,17,18, 21-26, 37, 38. Además, se demuestra de estos trabajos que en periodos cortos de tiempo la evaluación de la actividad hepatoprotectora de cualquier droga o fitomedicamento es más factible y productiva de realizar. 


\section{CONCLUSIÓN}

La actividad hepatoprotectora del extracto hidroalcohólico de Petroselinum crispum (Mill.) A.W. Hill (perejil) a dosis diarias de $150 \mathrm{mg} / \mathrm{kg}$ administrados por 30 días frente a la hepatotoxicidad inducida con etanol al 20\% durante 90 días en ratas albinas de la especie Holtzman es nula por lo encontrado en nuestros resultados. Sin embargo por los metabolitos secundarios del Petroselinum crispum podríamos rescatar que sus propiedades antiaoxidantes contribuyen con la prevención de los daños hepáticos y por lo tanto recomendamos que se desarrollen otros estudios experimentales al respecto incrementando la cantidad de etanol en la inducción y de las dosis de Petroselinum

\section{REFERENCIAS BIBLIOGRÁFICAS}

1. George Boon-Bee Goh, Wan-Cheng Chow, Renwei Wang, Jian-Min Yuan, Woon-Puay Koh. Coffee, alcohol and other beverages in relation to cirrhosis mortality: The Singapore Chinese Health Study .Hepatology, August, 2014. 10.1002/hep.27054 (http://onlinelibrary.wiley.com/ doi/10.1002/hep.27054/epdf).

2. Hyo-Jung Kwon, Young-Suk Won, Ogyi Park, Binxia Chang, Michae J. Duryee, Geoffrey E. Thiele, Akiko Matsumoto, Surendra Singh, Mohamed A. Abdelmegeed, Byoung-Joon Song, Toshihiro Kawamoto, Vasilis Vasiliou, Geoffrey M. Thiele, Bin Gao. Aldehyde dehydrogenase 2 deficiency ameliorates alcoholic fatty liver but worsens liver inflammation and fibrosis in mice. Hepatology, July, 2014. 10.1002/hep.27036 (http:// onlinelibrary.wiley.com/doi/10.1002/hep.27036/epdf).

3. Fuente de los datos: Informe Mundial de Situación sobre Alcohol y Salud 2014.

4. Musayon-Oblitas F. Potencial impacto social y económico de la muerte prematura por consumo de alcohol: Estimaciones en base a datos de mortalidad. Perú, 2008. Lima: Universidad Peruana Cayetano Heredia; 2009.

5. Fabián Fiestas; Reduciendo la carga de enfermedad generada por el consumo de alcohol en el Perú: propuestas basadas en evidencia. Rev. perú. med. exp. salud publica v.29 n.1 Lima mar. 2012.

6. Isau Huamantupa, Magaly Cuba, Rosa Urrunaga, Elías Paz, Nelson Ananya. Riqueza, uso y origen de plantas medicinales expendidas en los mercados de la ciudad del Cusco. 2011;18

7. Eduardo DELLACASSA SC, Arnaldo L. BANDONI, Salvador CAÑIGUERAL. Plantas Medicinales y Fitoterapia: ¿Indicadores de Dependencia o Factores de Desarrollo? Lat Am J Pharm. 2003 Mar 16;22:265-78.

8. A. Reyes-Munguía, D. Zavala-Cuevas, A. Alonso-Martínez. PEREJIL (PETROSELINUM CRISPUM): COMPUESTOS QUÍMICOS Y APLICACIONES. 2012 Diciembre;11. Available from: http://www.eumed.net/rev/ tlatemoani/11/perejil-compuestos-quimicos-aplicaciones.html. crispum administrada, prolongando el tiempo de inducción de la hepatotoxicidad crónica para describir la posible prevención de nódulos de regeneración y posterior cirrosis con algún fitomedicamento y a su vez sumar las investigaciones al respecto.

Financiamiento: Autofinanciado.

Conflicto de interés: Los autores declaran no tener conflictos de interés en la publicación de este artículo.

Recibido: 24 de octubre

Aprobado: 21 de diciembre
9. Nagamani Samundeeswari, Murugan Rajadurai, Palanisamy Ganapathy, Sukumaran Rexlin Shairibha. Effect of Vimliv on lipid profile and histopathology in ethanol-induced hepatotoxicity in albino Wistar rats. Int J Nutr Pharmacol Neurol Dis. 2013 Jun;

10. Gema Selema de la Morena, Jorge Martínez Pérez. EFECTO HEPATOPROTECTOR INDUCIDO POR EL FLAVONOIDE ASTILBINA FRENTE A UN MODELO ANIMAL TRATADO CON TETRACLORURO DE CARBONO. Rev Cubana Plant Med. 1999;1:36-9.

11. Elejalde Guerra Jl. Estrés oxidativo, enfermedades y tratamientos antioxidantes. An Med Interna. 2001 Jun;18(6):50-9.

12. Galicia-Moreno M, Gutiérrez-Reyes G. Papel del estrés oxidativo en el desarrollo de la enfermedad hepática alcohólica. Rev Gastroenterol México. 2014 Apr;79(2):135-44.

13. (CSIC) D de P del CS de IC. Valores hematológicos y bioquímicos de las ratas Sprague Dawley producidas en CENPALAB [Internet]. [cited 2015 Jul 24]. Available from: http://www.erevistas.csic.es/ficha_articulo. php?url=oai_revista68:2477\&oai_iden=oai_revista68

14. Porras N, Zeidán Q, Strauss M, Anselmi G. Valores Séricos de Marcadores Hepáticos en Ratas: Aporte de un Patrón de Referencia. Arch Venez Farmacol Ter. 2002 Jul;21(2):201-4.

15. Teixeira MA, Chaguri LC a. G, Carissimi AS, Souza NL de, Mori CMC, Gomes VMW, et al. Hematological and biochemical profiles of rats (Rattus novergicus) kept under microenvironmental ventilation system. Perfis hematol6gico e bioqufmico de ratos (Rattus norvegicus) mantidos sob sistema de ventilacao microambiental [Internet]. 2000 [cited 2015 Jul 25]; Available from: http://www.lume.ufrgs.br/handle/10183/99234

16. Víctor Hernán Arcila Quiceno MVZ, Esp. Comparación de los valores de referencia hematológicos en ratas wistar/UIS (Rattus norvergicus) con parámetros establecidos en laboratorios de altos estándares. REVISTA DE INVESTIGACIONES EN CIENCIAS ANIMALES - UNIVERSIDAD COOPERATIVA DE COLOMBIA» Volumen 6, N 12 [Internet]. 2010 Jun [cited 2015 Jul 25];6(12). Available from: http://wb.ucc.edu.co/sdmvz/ numeros/volumen-6-n-12/ 
17. Miquiel Planas, Mireia. Desarrollo y validación de un modelo experimental de encefalopatía hepática en ratas con ascitis. Efectos de la administración de IGF-1 frente a rifaximina en este modelo experimental [Internet]. [Barcelona]: Universidad autónoma de Barcelona; 2008. Available from: http://www.tdx.cat/bitstream/handle/10803/4523/ mmp1de1.pdf?sequence $=1$

18. Corredor OMA, Giménez E. Alteraciones enzimáticas hepáticas en ratas tratadas con vitamina E (a-tocoferol). MedULA. 2013;22(1):40-6.

19. Poveda E, Ayala P, Rodríguez M, Ordóñez E, Baracaldo C, Delgado W, et al. Effects of vegetal oil supplementation on the lipid profile of Wistar rats. Biomédica. 2005 Mar;25(1):101-9.

20. Dra. Aleida Herrera Batista, Dra. Maritza González Bravo, Dra. Ela Céspedes Miranda, Lic. Sonia Sánchez, González. EFECTOS DEL ALCOHOLISMO CRÓNICO SOBRE EL HÍGADO DE RATAS ALBINAS ADOLESCENTES. Rev Cubana Invest Biomed. 1999;189-96.

21. Troncoso L, Guija E. Efecto antioxidante y hepatoprotector del Petroselinum sativum (perejil) en ratas, con intoxicación hepática inducida por paracetamol. An Fac Med. 2013 Feb 27;68(4):333-43.

22. Guevara-Vásquez A, Rodríguez EM, Tello CM, Julca RY. EFECTO DEL INFUSO DE Petroselinum sativum SOBRE LA INSUFICIENCIA HEPÁTICA INDUCIDA EN Rattus norvegicus var. albinus. PHARMACIENCIA. 2014 Oct 23;2(1):39-47.

23. Arnao-Salas Al, Suárez-Cunza S, Trabucco-Ricaldi J, Cisneros-Chinchay $\mathrm{R}$, Elena-Rodrigo M. Efecto hepatoprotector del extracto acuoso de Smallanthus sonchifolius (yacón) en un modelo de intoxicación con acetaminofén. An Fac Med. 2012 Jul;73(3):239-44.

24. Ochoa C, Granda C, Chapoñan M, Borja R, Borjas P, Ortiz J, et al. EFECTO PROTECTOR DE PEUMUS BOLDUS EN RATAS CON TOXICIDAD HEPÁTICA INDUCIDA POR PARACETAMOL. Cienc E Investig Medico Estud Latinoam [Internet]. 2012 Feb 26 [cited 2015 Jul 25];13(1). Available from: http:// www.cimel.felsocem.net/index.php/CIMEL/article/view/159

25. Toledo DB-, Escobar-Román R, Boffill-Cárdenas M, BetancourtMorgado E, Correa II-, Cáceres BA-. Evaluación del potencial hepatoprotector de la Mentha piperita L previo a la inducción de hepatotoxicidad con acetaminofen [Internet]. Boletín Latinoamericano y del Caribe de Plantas Medicinales y Aromáticas. 2014 [cited 2015 Jul 25]. Available from: http://www.redalyc.org/articulo.oa?id=85632545005

26. Toledo DB, Cárdenas MB, Morgado EB, Román RE, Correa II, Cárdenas BA. Evaluación preclínica de la actividad hepatoprotectora de Ocimum basilicum L. y Allium sativum L. [Internet]. MediSur. 2014 [cited 2015 Jul 25]. Available from: http://www.redalyc.org/articulo.oa?id=180030081007

27. Troncoso L, Guija E, Bernui I, Cairo Y, Oriondo R, Pacheco A. Capacidad antioxidante de alimentos y plantas medicinales. Libro de resúmenes. Primer Congreso Internacional FITO 2000 y 1er Congreso Peruano de Plantas Medicinales y Fitoterapia. Lima: Instituto de Fitoterapia Americano; 2000

28. Troncoso L, Guija E, Oliveira G, Palomino F, Norberto V, Quiroz K. Evaluación toxicológica aguda del extracto acuoso y fracción $B$ (extracción con etanol y agua) del perejil (Petroselinum sativum) en ratas.
Libro de resúmenes. IV Jornadas Científicas Sanfernandinas y VII Jornadas de Investigación en Salud. An Fac Med. 2005;66 Supl 1:S26

29.Troncoso L, Guija E, Quiroz K. Capacidad antioxidante de la Beta vulgaris L. var. Crassa (Betarraga). Libro de resúmenes. I Congreso Internacional y VII Congreso Peruano de Nutrición "Alimentación y Nutrición para una Vida Saludable". Lima, Perú: Sociedad Peruana de Nutrición; 2004.

30. Troncoso L, Guija E, Quiroz K. Capacidad antioxidante del "Chimichurri" y sus componentes. Libro de resúmenes. Segundo Congreso Internacional FITO 2003 y 2do Congreso Peruano de Plantas Medicinales y Fitoterapia. Lima, Perú: Instituto de Fitoterapia Americano; 2003.

31. Troncoso L, Guija E, Quiroz K. Capacidad antioxidante de los condimentos del "Chimichurri". Libro de resúmenes. Segundo Congreso Internacional FITO 2003 y 2do Congreso Peruano de Plantas Medicinales y Fitoterapia. Lima, Perú: Instituto de Fitoterapia Americano; 2003.

32. Troncoso L, Guija E, Quiroz K. Efecto de la temperatura sobre la generación de radicales libres y la capacidad antioxidante del Capsicum annuum L. (Pimiento). Libro de resúmenes. Segundo Simposium Internacional de Plantas Medicinales y Fitoterapia. FITO 2004. Lima, Perú: Instituto de Fitoterapia Americano; 2004.

33. Troncoso L, Guija E, Quiroz K. Efecto de la temperatura sobre la capacidad antioxidante de la Allium cepa L. var. Aggregatum (Cebolla china). Libro de resúmenes. XXII Congreso Peruano de Química. Lima, Perú: Sociedad Química del Perú; 2004.

34. Troncoso L, Guija E, Ronceros S, Valdivia F, Flores J, Guija H y col. Efecto del camu-camu (Myrciaria dubia) y perejil (Petroselinum sativum) en plasma sanguíneo de pacientes con dislipidemia y diabetes mellitus tipo 2. Libro de resúmenes. III Jornadas de Investigación en Salud. Lima: Facultad de Medicina, UNMSM; 2001.

35. Troncoso L, Nolberto V, Oliveira G, Torrealva L, Guija H, Quiroz K. Estudio fitoquímico del Petroselinum sativum (perejil): Separación y caracterización de fracciones con capacidad antioxidante. Libro de resúmenes. III Jornadas Científicas Sanfernandinas y VI Jornadas de Investigación en Salud. An Fac Med. 2004;65 Supl 1:S20-21.

36. Dr. Ignacio A. Sisamón. ACERCA DE LA HEPATOTOXICIDAD DEL PARACETAMOL. Rev Hosp Priv Comunidad [Internet]. 2003;6. Available from: http://www.hpc.org.ar/images/revista/300-v6n2p42.pdf

37. ACTIVIDAD HEPATOPROTECTORA DEL EXTRACTO DE DIENTE DE LEÓN (Taraxacum officinale) EN RATAS (Rattus novergicus) CON HEPATOTOXICIDAD INDUCIDA POR TETRACLORURO DE CARBONO [Internet] [TESIS DE GRADO]. [RIOBAMBA Ecuador]: ESCUELA SUPERIOR POLITÉCNICA DE CHIMBORAZO FACULTAD DE CIENCIAS ESCUELA DE BIOQUÍMICA Y FARMACIA; 2012. Available from: http://dspace.espoch. edu.ec/bitstream/123456789/2590/1/56T00367.pdf

38. Jassim AM. Protective Effect of Petroselinum crispum(parsley)extract on histopathological changes in liver ,kidney and pancreas induced by Sodium Valproate- In male Rats. البيطرية الطبية للعلوم الكوفة مجلة Kufa J Vet Med Sci [Internet]. 2013 Jun 15 [cited 2015 Jul 25];4(1). Available from: http://www.uokufa.edu.iq/journals/index.php/kjvs/ article/view/1529 